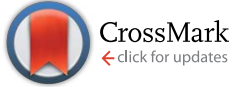

Cite this: RSC Adv., 2017, 7, 87

Received 5th October 2016 Accepted 28th November 2016 DOI: $10.1039 / c 6 r a 24745 h$

www.rsc.org/advances

\section{Aesthetically improved and efficient tannin-metal chelates for the treatment of dentinal hypersensitivity ${ }_{\dagger}^{\dagger}$}

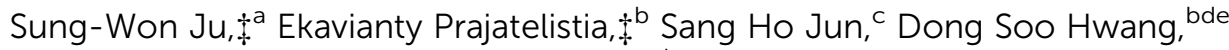 \\ Jin-Soo Ahn*a and Naresh D. Sanandiya*d
}

Dentinal hypersensitivity is an acute transient pain caused by the exposition of the dentinal tubules. The exposed tubules not only cause hypersensitivity but also cause the yellow coloration of the teeth due to their dominance in teeth coloration. An occlusion of the open dentinal tubules by the in situ biomineralization of hydroxyapatite ( $\mathrm{HAp}$ ) is so far the most effective method to alleviate the dentinal hypersensitivity. In recent years, several remineralization strategies, employing various substrates, have been studied for the treatment of dentinal hypersensitivity. However, aesthetically ideal solutions that include the tooth whitening aspect are poorly studied alongside with the tubular occlusion. Herein, the aesthetical improvement along with significant bioremineralization of the HAp crystals for the treatment of dentinal hypersensitivity was examined after a tannin (TA)-metal (ions and oxides) chelates mediated mineralization. Thus, demineralized human molar disks were treated with tannin-based chelates (TA-M). Briefly, TA-M denotes TA combined with 5 different daily intake metal ions and metal oxides, such as $\mathrm{Sr}\left(\mathrm{NO}_{3}\right)_{2}, \mathrm{Fe}_{2} \mathrm{O}_{3}, \mathrm{TiO}_{2}, \mathrm{CaCl}_{2}, \mathrm{VCl}_{3}$, and a non-metal oxide $\mathrm{SiO}_{2}$ in separate experiments. The samples were coated in the TA-M solution for 4 min followed by immersion in an artificial saliva for 7 days. Biomineralized HAp crystals were well-characterized by various analytical techniques. Among these, TA$\mathrm{Sr}\left(\mathrm{NO}_{3}\right)_{2}$ and $\mathrm{TA}-\mathrm{TiO}_{2}$ exhibited the most promising results, as they occluded ca. $79 \%$ and $68 \%$ of the dentinal tubules, respectively, with brighter color changes after the treatment. This desensitizing solution camouflaged the exposed dentin by its whitening effect using biocompatible $\mathrm{Sr}$ and $\mathrm{Ti}$ oxides, which may be potential for developing facile 'at-home' aesthetically effective dental desensitizer solution.

\section{Introduction}

Dentinal hypersensitivity, a common and elusive oral health problem in the world, has a prevalence of $\sim 15-57 \%$ in the general population and occurs when the dentin, which is usually overlaid by the enamel or cementum of a tooth, becomes exposed to the direct oral environment due to conditions such as bacterial demineralization, abrasion or gingival recession. ${ }^{1}$ Dentin, composed of $20 \%$ of type I collagen fibrils

${ }^{a}$ Dental Research Institute and Department of Biomaterials Science, School of Dentistry Seoul National University, Seoul 110-749, South Korea.E-mail: ahnjin@snu.ac.kr

${ }^{b}$ School of Interdisciplinary Bioscience and Bioengineering, Pohang University of Science and Technology, Pohang 790-784, South Korea

${ }^{c}$ Division of Oral and Maxillofacial Surgery, Department of Dentistry, Korea University Anam Hospital, Seoul 136-705, South Korea

${ }^{d}$ Division of Integrative Bioscience and Biotechnology, Pohang University of Science and Technology, Pohang 790-784, South Korea.E-mail: nareshsanandiya@gmail.com ${ }^{e}$ School of Environmental Science and Engineering, Pohang University of Science and Technology (POSTECH), Pohang 790-784, South Korea

† Electronic supplementary information (ESI) available. See DOI: 10.1039/c6ra24745h

† S. W. Ju and E. Prajatelistia contributed equally to this work. reinforced with $70 \%$ Hap and $10 \%$ plasma-like fluid, is a biomineralized hard tissue containing tiny capillaries called tubules. ${ }^{2}$ Inside each tubule lies a nerve branch that comes from the tooth pulp cavity. When the underlying dentin is exposed, thermal, tactile, osmotic or chemical stimuli (in the saliva flowing around the exposed dentinal tubules) provoke movement in the intradentinal fluid, which transmits to the afferent intradental nerves and results in the transient severe pain that is termed as dentinal hypersensitivity. Moreover, the color of the exposed dentin is more yellow than that of the enamel and it defines the overall teeth color. Therefore, occluding exposed dentinal tubules is proposed as a fundamental strategy for reducing the stimuli-evoked fluid moves to desensitize the dentin, as well as to reduce the yellowish staining of the teeth.

Procedures to cure or reduce dentinal hypersensitivity vary from 'at-home' self-administered desensitizing therapies to 'inoffice'-more complex clinical processes, which includes dental sealants or laser therapy by dental professionals in the dental office. However, to date, there is no universally satisfactory regimen that significantly cures and relieves the dentinal hypersensitivity. Over the decades, several attempts have been made to occlude the tubules by various commercially available 
remineralization systems. However, most of them contain high concentrations of glutaraldehyde, oxalate or acrylate as the active ingredient. ${ }^{3}$ Furthermore, these chemicals have been reported to show a high toxic effect on the human gingival fibroblasts, the most abundant structural cells in the periodontal tissues, therefore, they require a proper administration of the correct dosage, which means that they have to be administered by a clinician. ${ }^{4}$

In our continuous attempts to develop an efficient system for the treatment of dentinal hypersensitivity, bio-inspired catechol-metal complexation strategies have been studied that mimics the wound healing mechanism of the tunicates., Significant results have been obtained using gallic acid- $\mathrm{Fe}^{3+}$ as an active ingredient, achieving an $87 \%$ occlusion of the dentinal tubules after 7 days, ${ }^{7}$ and in the case of TA combined with $\mathrm{Fe}^{3+}$, a $\sim 47 \%$ occlusion of the dentinal tubules was achieved. ${ }^{8}$ Although previous reports offer potential applications, the high cost of gallic acid as well as the fact that iron ions were reported to form harmful insoluble hydroxide and phosphate precipitates at physiological and higher pHs led us to abandon this treatment. ${ }^{9}$ Tooth discoloration may be an impediment for the practical application since some metal complexes showed dentin stains after the treatment., ${ }^{\mathbf{8} 10}$

Tannin is a common, low-cost, and edible plant polyphenol present in red wine, fruits, tea and virtually in all families of plants. Tannin could be a promising candidate because its building blocks and trihydroxyphenyl containing molecules can be used as precursors for the formation of multifunctional coatings. ${ }^{11}$ In the presence of a mild aqueous solution of metal ions and metal oxides, many pyrogallol groups in TA are able to form chelates and adhere to various substrates within $30 \mathrm{~s}$, probably due to strong and reversible coordination bonds and cation- $\pi$ interactions in water. ${ }^{5,12-14}$ In addition, the pyrogallol groups of TA can strongly capture calcium ions, a key component of HAp, and supports the HAp nucleation, which leads to the occlusion of the dentinal tubules. ${ }^{8}$ However, a comprehensive study of tannic acid with different metal ions, metal oxides and non-metal oxides (e.g. $\mathrm{Sr}^{2+}, \mathrm{Ca}^{2+}, \mathrm{Fe}^{3+}, \mathrm{V}^{3+}, \mathrm{TiO}_{2}, \mathrm{Fe}_{2} \mathrm{O}_{3}$, and $\mathrm{SiO}_{2}$ ), as well as the aesthetic improvement of the tannin-metal chelates, have not been studied, concerning their effects on the dentinal occlusion and practical application.

The aim of the present study was to disclose the major sources and explore this low-cost plant polyphenol, concerning its chelation with various metal ions and metal oxides for the biomineralization of Hap, which cures the dentinal hypersensitivity treatment, as well as study the aesthetic evolution by measuring the color changes after the treatment. The comprehensive data set of TA-M for dentinal hypersensitivity treatment along with the aesthetic improvement has been furnished for their potential applications.

\section{Materials and methods}

\subsection{Materials}

Tannic acid, $\mathrm{Sr}\left(\mathrm{NO}_{3}\right)_{2}, \mathrm{TiO}_{2}$ (particle size: $21 \mathrm{~nm}$ ), $\mathrm{SiO}_{2}$ (particle size: $100 \mathrm{~nm}), \mathrm{Fe}_{2} \mathrm{O}_{3}, \mathrm{CaCl}_{2}, \mathrm{VCl}_{3}$, antibiotic mixtures, and trypsin were purchased from Sigma-Aldrich (St. Louis, MO,
USA). $\mathrm{KH}_{2} \mathrm{PO}_{4}$ was purchased from Samchun (Seoul, S. Korea) and tris(hydroxymethyl)aminomethane was purchased from EM Science (Gibbstown, NJ, USA). DMEM and FBS were purchased from Hyclone (UT, USA). All chemicals were of analytical grade and were used as received unless otherwise noted.

\subsection{Preparation of the acid-etched tooth slices}

All experiments were performed in compliance with the relevant laws or guidelines. The guidelines of the institutional review board (IRB AN14259-001) of the Korea University Medical Centre (Seoul, Korea) were followed to obtain human molars. Besides, informed consent was obtained from all subjects. The tooth slices from human molars were treated with $3 \%$ sodium hypochlorite solution to sterilize these from bacteria and rinsed with phosphate buffered saline (PBS). Dentin samples were taken from the tooth crown and were perpendicularly sliced to the longitudinal axis into $1 \mathrm{~mm}$ thick disks with a diameter of approximately 8-10 mm using a low-speed diamond saw blade. The tooth slices were then acid-etched with $3 \% \mathrm{HNO}_{3}$ solution for $1 \mathrm{~min}$ followed by rinsing with sufficient deionized water.

\subsection{Coating and remineralization methods}

Six different aqueous solutions of $\mathrm{Sr}\left(\mathrm{NO}_{3}\right)_{2}, \mathrm{SiO}_{2}, \mathrm{Fe}_{2} \mathrm{O}_{3}, \mathrm{TiO}_{2}$, $\mathrm{CaCl}_{2}$, and $\mathrm{VCl}_{3}$ at a $1.2 \mathrm{mM}$ concentration were freshly prepared for separate experiments. TA $(0.47 \mathrm{mM})$ and metal ion solutions were vigorously mixed and the $\mathrm{pH}$ was raised to 8.0 by slowly adding $\mathrm{NaOH}(0.1 \mathrm{M})$ solution. The tooth slices were immersed in a TA-M solution for $4 \mathrm{~min}$, changing the solution after 1 minute intervals. Then, these tooth slices were immersed in deionized water and used wherever applicable.

The calcification solution, herein referred to as artificial saliva, was prepared following the previously reported procedure. Briefly, the solution containing calcium $\left(\mathrm{CaCl}_{2} \cdot 2 \mathrm{H}_{2} \mathrm{O}, 8\right.$ $\mathrm{mM})$, phosphate $\left(\mathrm{KH}_{2} \mathrm{PO}_{4}, 1.55 \mathrm{M}\right)$, fluoride $\left(\mathrm{NaF}, 1 \mathrm{mg} \mathrm{L}{ }^{-1}\right)$, and $\mathrm{NaCl}(180 \mathrm{mM})$ was buffered with tris(hydroxymethyl)aminomethane- $\mathrm{HCl}(50 \mathrm{mM})$. The $\mathrm{pH}$ of the calcification solution was adjusted to $7.6 \mathrm{using} \mathrm{HCl}(0.1 \mathrm{~N})$ and $\mathrm{NaOH}(0.1 \mathrm{M})$ solutions and stored at $4{ }^{\circ} \mathrm{C}$ prior to use. Tooth slices with and without coating were immersed in the calcification solution (10 $\mathrm{mL}$ ) at the bottom of the polyethylene tubes. All samples were placed in a shaking incubator at $100 \mathrm{rpm}$ and $37^{\circ} \mathrm{C}$, and the calcification solution was refreshed every day. The tooth slices were removed after 7 days, cleaned by ultrasonication in deionized water, and rinsed several times with deionized water, followed by drying at room temperature prior to characterizations.

\subsection{Characterizations of the precipitates of remineralization}

The surface morphologies of the control and treated dentin samples, following a 4 min treatment and subsequent 7 days remineralization treatment in the artificial saliva solution, were analyzed using a field emission scanning electron microscopy (FE-SEM, JEOL, Tokyo, Japan) at $10 \mathrm{kV}$. The average diameters and lengths of the HAp nanorods in the composite were determined by transmission electron microscopy (TEM, JEOL) 
and SEM using ImageJ software. The diffraction pattern was observed using the selected area electron diffraction (SAED).

\subsection{Dentinal fluid permeability assay}

Dentinal hypersensitivity originates from the neural stimulation by the fluid flow within the dentinal tubules that is driven by a physiological pulpal pressure of approximately $200 \mathrm{~mm}$ $\mathrm{H}_{2} \mathrm{O}$. An in vitro dentinal fluid permeability assay was performed using a fluid filtration system according to our previous study to evaluate the TA-M induced biomineralization of Hap. Briefly, the amount of water infiltration in an untreated tooth slice for 10 min at a $200 \mathrm{~mm} \mathrm{H}_{2} \mathrm{O}$ pressure was first measured as the standard amount. The water infiltration amounts after the treatment with TA-metal for $4 \mathrm{~min}$, followed by incubation in the artificial saliva for 7 days, were again measured. The permeability of each sample was expressed as a percentage (\%) of the fluid flow. All experiments were carried out in triplicates with three independent samples.

\subsection{Colorimetric measurements}

To evaluate the color change induced by the TA-metal treatment, colorimetric measurements of the samples were performed using a reflectance colorimetric device (Spectroradiometer (PR-670), SpectraScan, Photo Research, Chatsworth, CA, USA). This device was set to produce color parameters based on the average daylight (D65: $6404 \mathrm{~K}$ ). The optical geometry of this system consists of a 45-degree illumination angle and 0-degree viewing angle to measure an area 3 $\mathrm{mm}$ in diameter. The color parameters were determined in the $L^{*} a^{*} b^{*}$ color space, as established by the Commission Internationale de l'Eclairage (CIELAB) in 1976. The mean of the results was determined by three separate successive measurements. Color differences $(\Delta E)$ were calculated according to eqn (1) and whiteness index $(W)$ was calculated according to eqn (2), as reported in the literature: ${ }^{15,16}$

$$
\begin{gathered}
\Delta E_{\left(L^{*}, a^{*}, b^{*}\right)}=\left[\left(L_{0}^{*}-L_{1}^{*}\right)^{2}+\left(a_{0}^{*}-a_{1}^{*}\right)^{2}+\left(b_{0}^{*}-b_{1}^{*}\right)^{2}\right]^{1 / 2} \\
W=100-\sqrt{ }\left(\left(100-L^{*}\right)^{2}+\left(a^{*}\right)^{2}+\left(b^{*}\right)^{2}\right)
\end{gathered}
$$

where $L^{*}$ is a group of lightness, $a^{*}$ is a group of red varying set, and $b^{*}$ is a group of yellow varying set. The samples were tested before treatment (denoted as 0 ), after the 4 min treatment (denoted as 1), and after the 7 days remineralization treatment (denoted as 2).

\subsection{Human periodontal ligament (hPDL) cell culture}

To evaluate the direct cytotoxicity and physiological adaptation of the human periodontal ligament (hPDL) cells, the 4th cell passage cells was examined. Proliferation was measured in three types of culture dishes: uncoated, $\mathrm{TA} / \mathrm{Sr}^{2+}$-coated, and remineralized sample using essential medium-alpha (MEM- $\alpha$; Hyclone, USA) media supplemented with $10 \%$ (v/v) fetal bovine serum (FBS; Hyclone) and 1\% penicillin/streptomycin (Hyclone) at $37{ }^{\circ} \mathrm{C}$ in a humidified atmosphere of $5 \% \mathrm{CO}_{2}$ and $95 \%$ air.
Subconfluent cells were detached using $0.25 \%$ trypsin-EDTA (Hyclone), and then viable cells were identified by the trypan blue assay and counted using a hemocytometer. After this, $5 \times$ $10^{4}$ cells ( $>95 \%$ viability) in MEM- $\alpha$ with $10 \%$ FBS were placed on each sample-coated glass disk, which was put in a 24-well plate. To count the number of viable cells, a Cell Counting Kit-8 solution (CCK-8; Dojindo Laboratories, Japan) was used; this solution produces a yellow formazan dye in the presence of viable cells. One to three days after the cell seeding, $50 \mu \mathrm{L}$ of CCK-8 was added to the wells for $3 \mathrm{~h}$ at $37{ }^{\circ} \mathrm{C}$ to allow the formation of formazan crystals, and then the absorbance was measured at $450 \mathrm{~nm}$ using a microplate reader (Bio-Rad).

\section{Results and discussions}

\subsection{Pyrogallol-mediated HAp remineralization}

Hydroxyl groups of TA initially participate to conjugate with the metal ions and metal oxides and incorporate the coating layer on the dentinal tubule. ${ }^{17}$ The assumed hypothesis is that some of the unconjugated hydroxyl groups of TA may interact with calcium ions of artificial saliva and form chelates, thereby inducing the HAp remineralization. ${ }^{18}$ On the other hand, TA-M may also interact with the organic interface of the dentin, thereby stabilizing the exposed collagen matrix. This interaction is an essential process in the cell adhesion and growth. ${ }^{19}$ Generally, TA-M potentially induce cross-linking in the dentinal collagen and grow densely-packed and well-aligned rod-like remineralized HAp crystals. These remineralized denselypacked HAp crystals hinder the osmotic fluid exchange with the internal dentinal nerves and prevent the stimulation of the dentinal nerves, which leads to a relief from the dentinal hypersensitivity. A schematic for the pyrogallol-mediated HAp remineralization and dentinal tubule occlusion is shown in Fig. 1.

\subsection{Characterizations}

To evaluate the surface deposits and topology of the tubular occlusion after treatment with TA-metal, treated and untreated dental disks were examined from the top and cross-section view by SEM (Fig. 2). The untreated sample showed well-defined and regular tubules (2-4 $\mu \mathrm{m}$ diameters) that penetrated the dentin perpendicularly to the surface (Fig. 2a). After 4 min of TA-metal treatment, it was observed that the tubule entrances had narrowed down although the middle of the tubules remained unchanged. This result indicates that the TA-M forms films that deposit onto the surface within $4 \mathrm{~min}$. After 7 days of remineralization in the artificial saliva, all tubules were significantly filled with needle-like HAp crystals (Fig. 2b-g). Although the human body has a natural self-remineralization ability, the process is very slow, which is in good agreement with the SEM image of the untreated sample.

Thus, considering the low concentration $(<0.016 \%)$ of the TA-metal used for this coating, the TA-metal coating shows an efficient deposition on the dentin compared to the commercial desensitizer studied in the previous report. ${ }^{8}$ Furthermore, the mechanical stability of the biomineralized HAp was checked for 

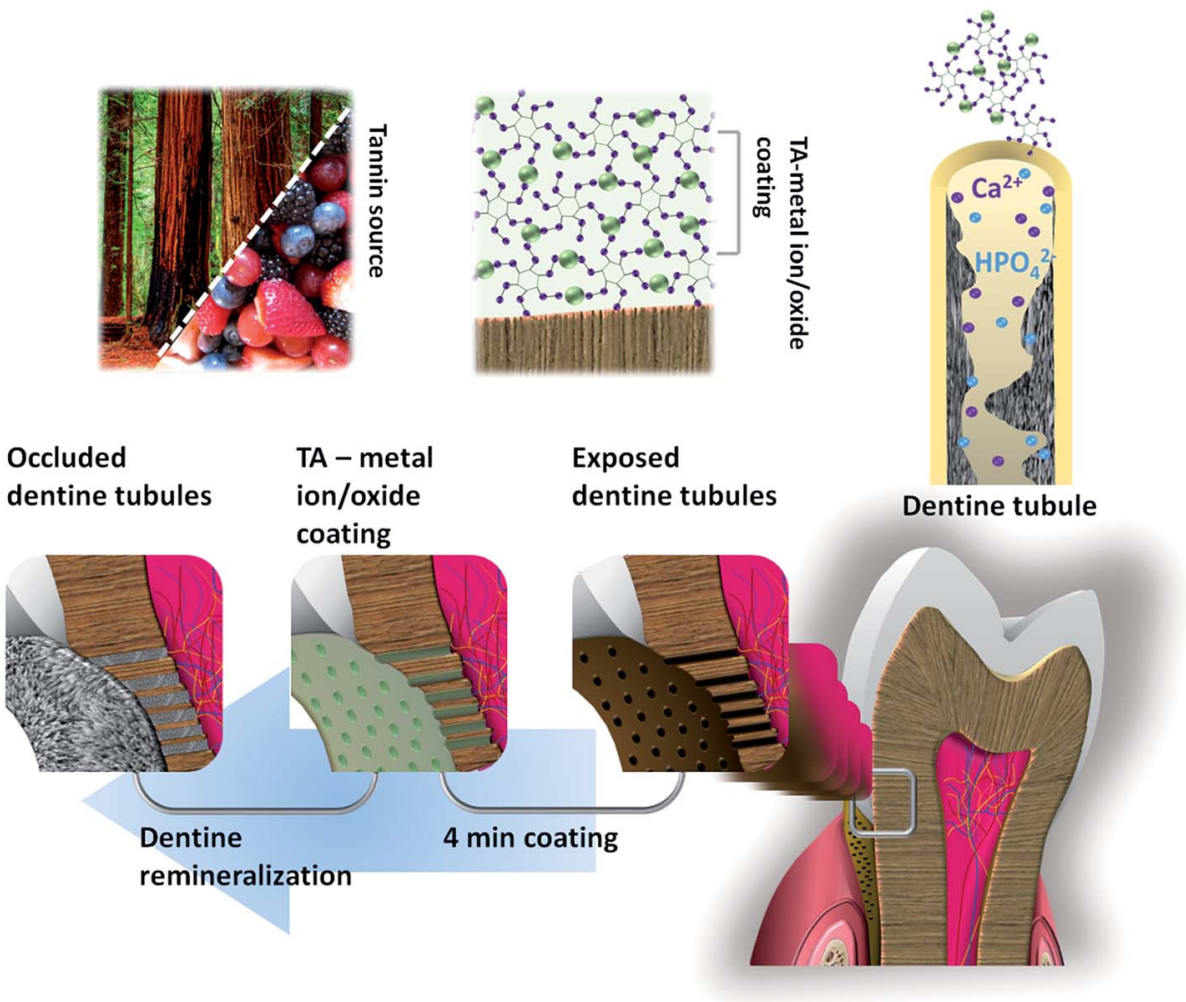

Fig. 1 Schematic of the TA-M-mediated occlusion of the exposed dentinal tubules for dentinal desensitizing.

the samples after the 7 day treatment, and it was stable after vigorous mechanical brushing for over 1000 strokes (Fig. S1†). This good mechanical stability can be well-explained by the fact that the pyrogallol groups have strong adhesion strength on the substrate and high density of cross-links with the metal ions/ oxides, similar to the natural behavior in tunicates, where they glue their torn tissues under the sea via pyrogallolmediated cross-linking. The results obtained herein are favourable for the oral environment, which is under various mechanical stresses such as chewing, brushing, and temperature-driven air pressure.

The needle-like HAp crystals deposited on the tubule surfaces were characterized by TEM and EDX (Fig. 3). The mineral-like material was fragmented in ethanol by ultrasonication for $30 \mathrm{~min}$. The EDX analysis revealed that the $\mathrm{Ca} / \mathrm{P}$ ratio of the needle-like crystals was 1.61 , having $100-300 \mathrm{~nm}$ length and 10-20 aspect ratio. The selected area electron diffraction (SAED) pattern exhibited characteristic peaks attributable to the (002), (004), (112), (211), and (300) planes, which are similar to the peaks found in the HAp pattern, and confirmed the crystals as HAp. ${ }^{20}$ The high-resolution TEM image clearly showed that the (002) crystal plane is perpendicular to the $c$-axis, a longitudinal axis of HAp, suggesting that an anisotropic growth of the needle-like HAp crystals occurred along the (002) plane, which is similar to that reported in the literature. ${ }^{20,21}$ The absence of HAp crystals in the untreated sample suggested the involvement of the pyrogallol groups in the HAp nucleation.
This result further confirmed that the pyrogallol groups initiate the TA-mediated anisotropic HAp remineralization. Pyrogallol groups in TA strongly capture calcium ions, which are the key components of HAp. ${ }^{22}$ Overall, pyrogallol groups of TA contributed in the adhesion to the substrate, mechanical reinforcement by forming $\mathrm{TA}-\mathrm{M},{ }^{23}$ and a highly anisotropic HAp formation by $\mathrm{Ca}^{2+}$ ion adsorption from the saliva. The highly anisotropic HAp has been considered to have a good bioreactivity and bioabsorbability. In addition, the mechanical properties of the naturally occurring HAp-embedded materials are modulated by the aspect ratio and arrangements of the HAp crystals.

\subsection{Dentinal fluid test and topological occlusion of the dentinal tubules}

The TA-metal coating was expected to function as a dentinal sealant and block the open dentinal tubules. To evaluate the occlusion effect of the TA-metal coating, dental disks prepared from human molar disks were used to measure the fluid permeability through the dentin. Permeability is derived from the hydraulic conductance or ease of the fluid flow through the dentin (Fig. S2 $†$ ). The TA-metal treatment blocked the water infiltration and results are depicted in Fig. 4b. TA with $\mathrm{TiO}_{2}$, $\mathrm{Sr}^{2+}, \mathrm{Fe}_{2} \mathrm{O}_{3}, \mathrm{Ca}^{2+}, \mathrm{SiO}_{2}, \mathrm{~V}^{3+}$, and $\mathrm{Fe}^{3+}$ blocked 29, 12, 38, 10, 7, 11 , and $33 \%$ of the tubules after the 4 min treatment, respectively, and $68,79,68,58,34,68$, and $37 \%$ after the 7 day treatment, respectively. The water infiltration result for TA$\mathrm{Fe}^{3+}$ was reproduced from a previous study for comparison in 
$4 \mathrm{~min}$
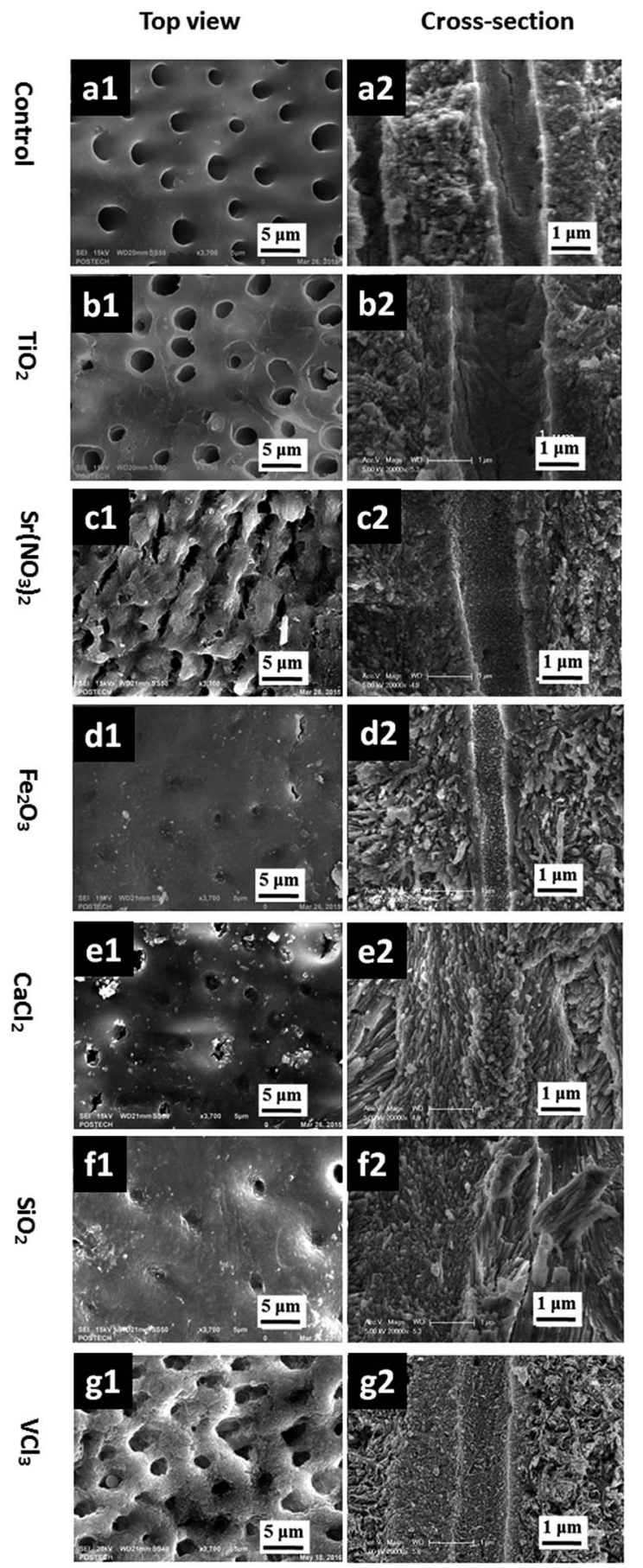

Cross-section
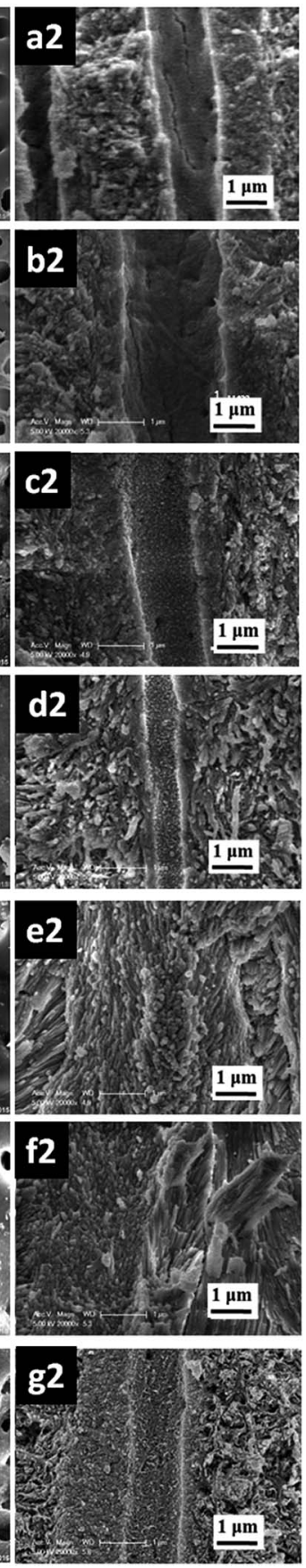

7 days

Top view
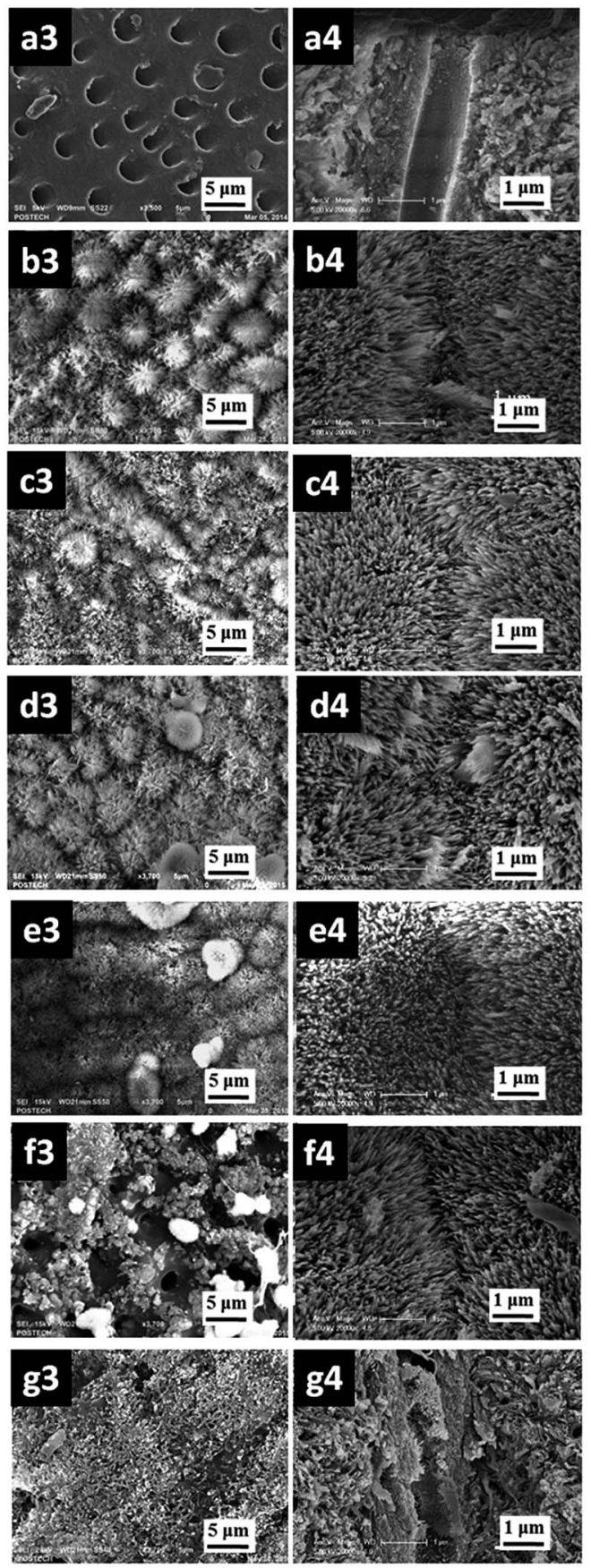

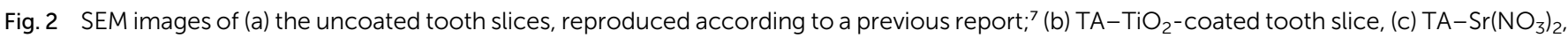
(d) $\mathrm{TA}-\mathrm{Fe}_{2} \mathrm{O}_{3}$, (e) $\mathrm{TA}-\mathrm{CaCl}_{2}$, (f) $\mathrm{TA}-\mathrm{SiO}_{2}$, and (g) $\mathrm{TA}-\mathrm{VCl}_{3}$-coated tooth slices; suffix denotes: (1) top view and (2) cross-section view after 4 min treatment in the respective TA-metal ion/oxide solution; (3) top view and (4) cross-section view after a 7 day immersion in the artificial saliva.

this study. ${ }^{8}$ For control TA, no dentinal occlusion after 4 min and the 7 day remineralization treatment was observed. ${ }^{8}$ The result suggests that the topologically observed TA-M actually restrained the dentinal fluid flow and the regenerated HAp significantly provided additional fluid blockage on the dentinal tubules.
The greater nucleation of HAp with $\mathrm{Sr}^{2+}$ ions may be explained by the fact that the human body absorbs strontium as if it was calcium due to the chemical resemblance of the elements. Interestingly, these serendipitous results are very encouraging because $\mathrm{Ti}$ and $\mathrm{Sr}$ ions exhibit a good biocompatibility and tissue compatibility. Strontium ions promote early 

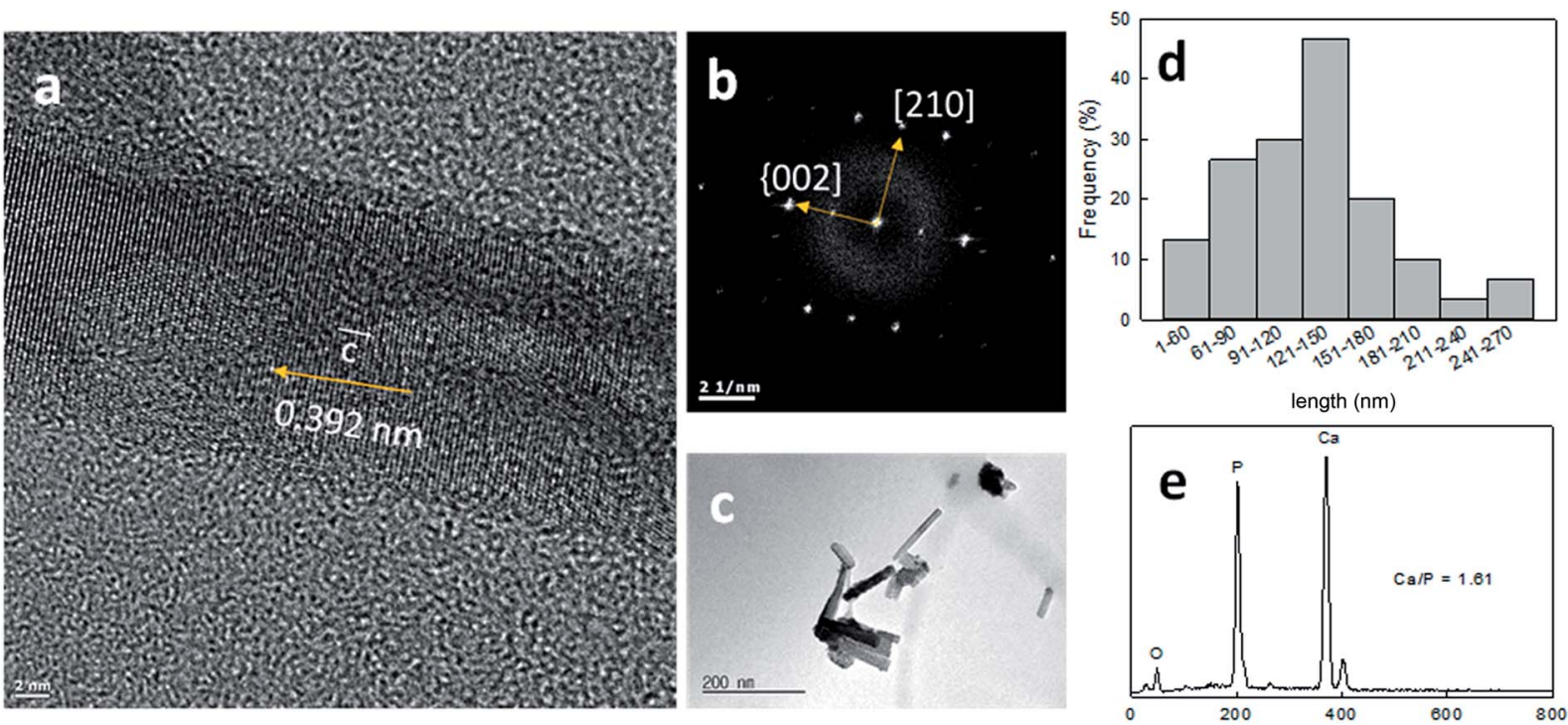

Fig. 3 Pyrogallol-mediated hydroxyapatite remineralization (a) HR-TEM image of the fragmented anisotropic crystals from a HAp-mineralized $\mathrm{TA} / \mathrm{Sr}^{2+}$-coated tooth slice; (b) selected area electron diffraction (SAED) pattern; (c) fragmented HAp crystal TEM image; (d) histogram with the HAp particle length distribution; and (e) EDX spectrum of the HAp-mineralized GA/Fe ${ }^{3+}$-coated tooth slice; Ca/P = calcium/phosphorus aspect ratio.

bone formation and its inherent ability for osseointegration has elicited a prominent clinical interest. ${ }^{24,25}$ On the other hand, $\mathrm{TiO}_{2}$ is considered as a promising bone scaffolding material because it promotes osteoblast adhesion, which induces bone formation, and its ability to osseointegrate, enabling its use in dental implants. ${ }^{26}$

\subsection{Color changes and whiteness index measurements}

Tooth staining after the therapy is an important aesthetic concern in clinical dentistry. The staining of the dentin was quantified by measuring the color change $(\mathrm{d} E)$ and whiteness index $(W)$ of the dentin after the TA-metal treatment (Fig. 5). The color change results after the TA-metal chelate treatment are presented in Fig. 5a, which shows that most of the TA-M used herein did not exceed the acceptability limit $(\mathrm{d} E=5.5)$, except for $\mathrm{TiO}_{2}$ and $\mathrm{SiO}_{2}$. The higher $\mathrm{d} E$ values for $\mathrm{TiO}_{2}$ and $\mathrm{SiO}_{2}$ were observed due to their higher whiteness indices (Fig. 5b), which makes them good candidates as tooth whitening additives. ${ }^{27}$ Dentin is covered by enamel or gum (gingiva), which by abrasion of enamel, dental caries or recession of gingiva causes the exposure of dentin. Usually, dentin has a more yellowish color and higher chroma than that of enamel; therefore, exposed dentin is responsible for both hypersensitivity and undesirable color change in teeth. ${ }^{28}$

The whiteness indices for $\mathrm{TA}-\mathrm{TiO}_{2}$ and $\mathrm{TA}-\mathrm{SiO}_{2}$ are greater (>60) than that for the rest of the chelates (Fig. 5b), and they also exhibited greater dentinal tubule occlusion (vide supra Section 3.3); therefore, $\mathrm{TA}-\mathrm{TiO}_{2}$ and $\mathrm{TA}-\mathrm{Sr}^{2+}$ chelates can be considered as potential candidates for the dentinal hypersensitivity
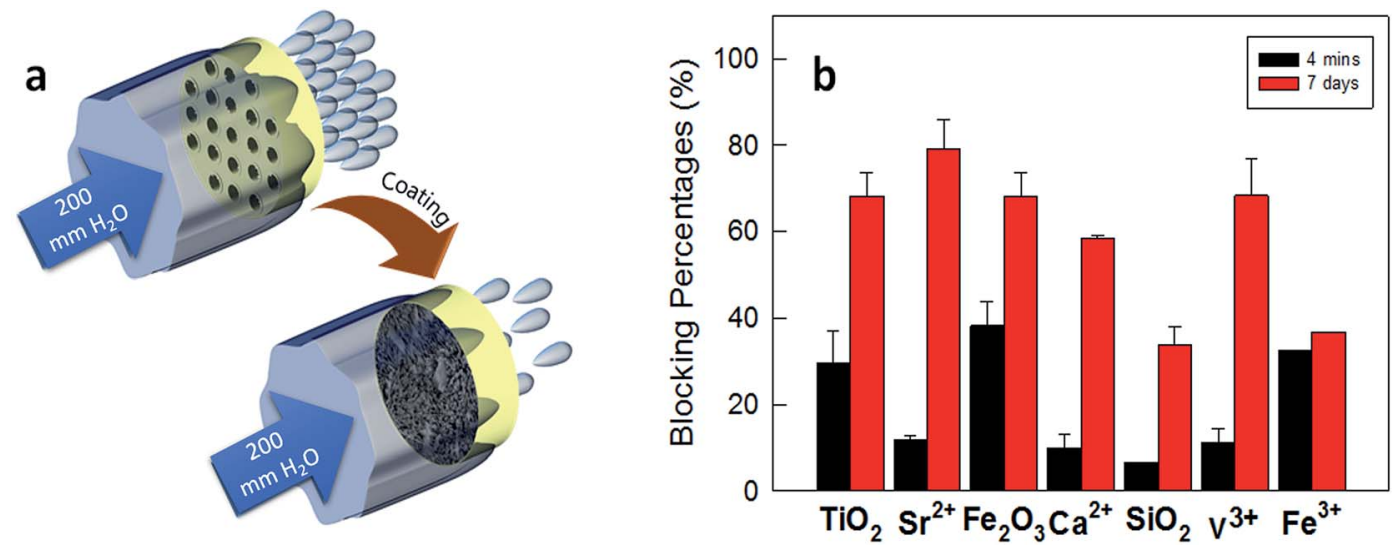

Fig. 4 Dentinal fluid flow tests: (a) evaluation of dentinal tubule occlusion. Water infiltration amount in (b) the TA-metal-coated tooth slices at $200 \mathrm{~mm} \mathrm{H} \mathrm{m}_{2} \mathrm{O}$ for $600 \mathrm{~s}$. Each value represents the mean of three analyses and its standard deviation; the TA-Fe ${ }^{3+}$ data used in this graph are reproduced according to our previous report. ${ }^{8}$ 

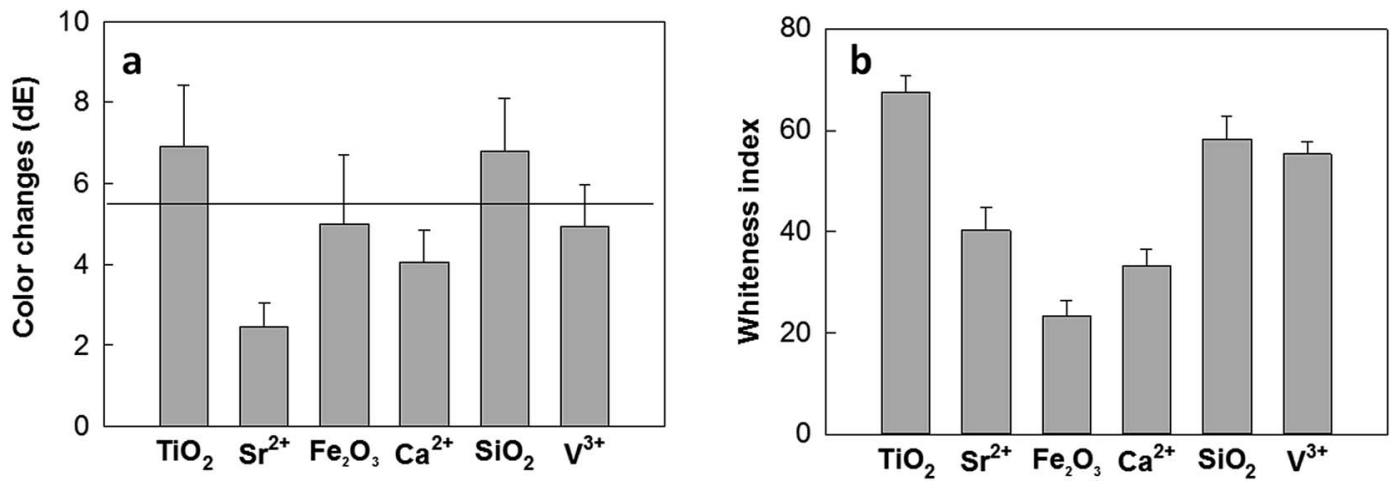

Fig. 5 (a) Color changes in the tooth slices by the TA-M treatment. The line is the acceptability guideline; (b) whiteness index of the TA-M coated samples.

treatment. This study provides a potential application of TAmetal chelates due to the fact that color changes to a brighter hue are desirable in terms of aesthetic improvement, which would offer an additional advantage to the hypersensitivity treatment in dentistry.

\subsection{Cell proliferation of the human periodontal ligament} (hPDL) cells

Tannin has been introduced to human and animal as an ingredient of food and tea since the origin of the life and thus its safety has already been proven..$^{29}$ To quantitatively evaluate the cytotoxicity of the TA-metal ion coating towards a human periodontal ligament cell (hPDL), cell proliferation after a $4 \mathrm{~min}$ coating with $\mathrm{TA}-\mathrm{Sr}^{2+}$ and 7 day remineralization were monitored for 3 days with a colorimetric assay and empty wells were used as a control (Fig. 6). The cell proliferation test showed viable cell numbers for the wells coated with $\mathrm{TA}-\mathrm{Sr}^{2+}$ only and moderately increased cell viability for the wells that underwent coating with $\mathrm{TA}-\mathrm{Sr}^{2+}$ plus remineralization. Hence, $\mathrm{TA}-\mathrm{Sr}^{2+}$, the optimized candidate of this study, proved low cytotoxicity towards the hPDL cells.

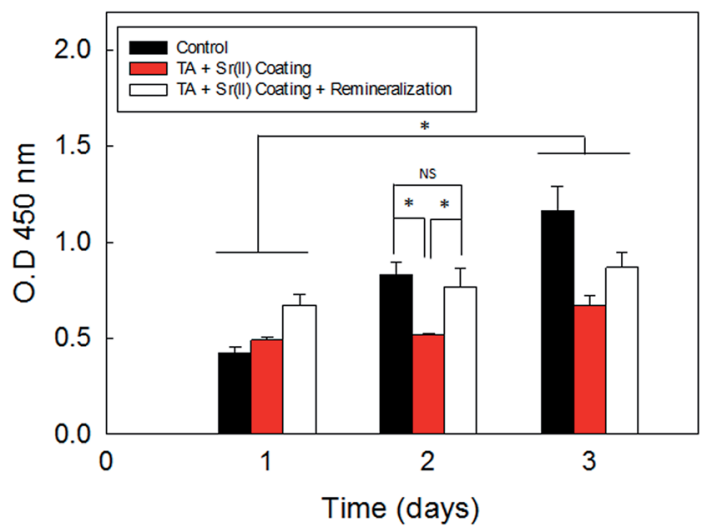

Fig. 6 Evaluation of the human periodontal ligament (hPDL) cell proliferation on the $\mathrm{TA} / \mathrm{Sr}^{2+}$-coated cell culture dish and hydroxyapatite remineralized cell culture dish $(n=3$; the mean \pm the standard error of the mean). NS = not significant $(P>0.05) . * P<0.05$.

\section{Conclusions}

In this study, we proved that the TA-metal coating occluded the exposed dentinal tubules by directly narrowing down the tubule entrances via induced HAp remineralization in the artificial saliva. At low concentration $(<0.016 \%)$, the TA- $\mathrm{Sr}\left(\mathrm{NO}_{3}\right)_{2}$ and TA$\mathrm{TiO}_{2}$ coatings were efficient and facile to occlude the tubules with an improved aesthetic effect after the treatment of dentinal hypersensitivity. The TA-metal formed a mechanically stable complex film on the microsized tubules in only $4 \mathrm{~min}$ and nucleated the densely packed HAp crystals after 7 days remineralization, which resulted in $\sim 79 \%$ occlusion in the case of $\mathrm{TA} / \mathrm{Sr}^{2+}$ chelate. This study may be potentially useful to develop sustainable, cost-competitive, and biocompatible products, such as toothpaste and mouthwash, with an aesthetically effective dental desensitizer solution.

\section{Acknowledgements}

Authors gratefully acknowledge the Marine Biotechnology program (Marine BioMaterials Research Center) funded by the Ministry of Oceans and Fisheries of Korea (D11013214H480000110), and the National Research Foundation of Korea Grant funded by the Korean Government (NRF-2013R1A1A1065373).

\section{References}

1 C. H. Splieth and A. Tachou, Clinical Oral Investigations, 2013, 17, 3-8.

2 M. Goldberg, A. B. Kulkarni, M. Young and A. Boskey, Front. Biosci., Elite Ed., 2011, 3, 711-735.

3 P. Schupbach, F. Lutz and W. J. Finger, Eur. J. Oral Sci., 1997, 105, 414-421.

4 A. Sengun, S. Buyukbas and S. S. Hakki, J. Biomed. Mater. Res., Part B, 2006, 78, 131-137.

5 H. Zeng, D. S. Hwang, J. N. Israelachvili and J. H. Waite, Proc. Natl. Acad. Sci. U. S. A., 2010, 107, 12850-12853.

6 D. X. Oh, S. Kim, D. Lee and D. S. Hwang, Acta Biomater., 2015, 20, 104-112.

7 E. Prajatelistia, S.-W. Ju, N. D. Sanandiya, S. H. Jun, J.-S. Ahn and D. S. Hwang, Adv. Healthcare Mater., 2016, 5, 919-927. 
8 D. X. Oh, E. Prajatelistia, S.-W. Ju, H. J. Kim, S.-J. Baek, H. J. Cha, S. H. Jun, J.-S. Ahn and D. S. Hwang, Sci. Rep., 2015, 5, 10884.

9 A. T. Iffat, Z. T. Maqsoodahida and N. Fatima, J. Chem. Soc. Pak., 2005, 27, 174-177.

10 K. G. Adcock and S. M. Hogan, The Journal of Pediatric Pharmacology and Therapeutics, 2008, 13, 162-165.

11 T. S. Sileika, D. G. Barrett, R. Zhang, K. H. A. Lau and P. B. Messersmith, Angew. Chem., Int. Ed., 2013, 52, 1076610770.

12 H. Ejima, J. J. Richardson, K. Liang, J. P. Best, M. P. van Koeverden, G. K. Such, J. Cui and F. Caruso, Science, 2013, 341, 154-157.

13 D. A. Dougherty, Science, 1996, 271, 163-168.

14 Q. Lu, D. X. Oh, Y. Lee, Y. Jho, D. S. Hwang and H. Zeng, Angew. Chem., Int. Ed., 2013, 52, 3944-3948.

15 R. D. Douglas and J. D. Brewer, J. Prosthet. Dent., 1998, 79, 254-260.

16 W. Luo, S. Westland, R. Ellwood, I. Pretty and V. Cheung, J. Dent., 2009, 37, 21-26.

17 A. Nakajima and T. Sakaguchi, J. Chem. Technol. Biotechnol., 1993, 57, 321-326.

18 S. Kim, S. Don and D. E. Mainwaring, J. Appl. Polym. Sci., 1997, 65, 1795-1805.

19 P. Velmurugan, E. R. A. Singam, R. R. Jonnalagadda and V. Subramanian, Biopolymers, 2014, 101, 471-483.
20 J. Ryu, S. H. Ku, H. Lee and C. B. Park, Adv. Funct. Mater., 2010, 20, 2132-2139.

21 Y.-Z. Zhou, Y. Cao, W. Liu, C. H. Chu and Q.-L. Li, ACS Appl. Mater. Interfaces, 2012, 4, 6901-6910.

22 J. Guo, Y. Ping, H. Ejima, K. Alt, M. Meissner, J. J. Richardson, Y. Yan, K. Peter, D. von Elverfeldt, C. E. Hagemeyer and F. Caruso, Angew. Chem., Int. Ed., 2014, 53, 5546-5551.

23 W. M. Chirdon, W. J. O'Brien and R. E. Robertson, J. Biomed. Mater. Res., Part B, 2003, 66, 532-538.

24 O. Z. Andersen, V. Offermanns, M. Sillassen, K. P. Almtoft, I. H. Andersen, S. Sørensen, C. S. Jeppesen, D. C. E. Kraft, J. Bøttiger, M. Rasse, F. Kloss and M. Foss, Biomaterials, 2013, 34, 5883-5890.

25 M. Schumacher and M. Gelinsky, J. Mater. Chem. B, 2015, 3, 4626-4640.

26 N. Wang, H. Li, W. Lü, J. Li, J. Wang, Z. Zhang and Y. Liu, Biomaterials, 2011, 32, 6900-6911.

27 E. El-Meliegy and R. v Noort, Glasses and Glass Ceramics for Medical Applications, Springer, New York, 2012.

28 J. J. ten Bosch and J. C. Coops, J. Dent. Res., 1995, 74, 374380.

29 J. H. Park, K. Kim, J. Lee, J. Y. Choi, D. Hong, S. H. Yang, F. Caruso, Y. Lee and I. S. Choi, Angew. Chem., Int. Ed., 2014, 53, 12420-12425. 